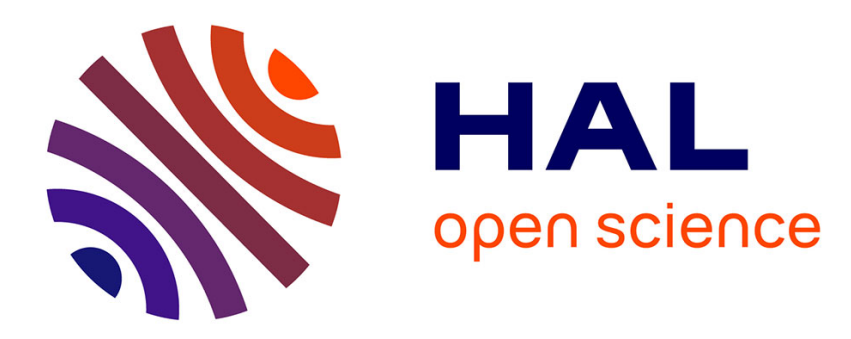

\title{
Homogenization of a highly rarefied binary structure of finite diffusivity
}

Isabelle Gruais, Dan Polisevski

\section{To cite this version:}

Isabelle Gruais, Dan Polisevski. Homogenization of a highly rarefied binary structure of finite diffusivity. Applicable Analysis, 2007, 86 (5), pp.633-652. 10.1080/00036810701354979 . hal-00364510

\author{
HAL Id: hal-00364510 \\ https://hal.science/hal-00364510
}

Submitted on 11 Dec 2017

HAL is a multi-disciplinary open access archive for the deposit and dissemination of scientific research documents, whether they are published or not. The documents may come from teaching and research institutions in France or abroad, or from public or private research centers.
L'archive ouverte pluridisciplinaire HAL, est destinée au dépôt et à la diffusion de documents scientifiques de niveau recherche, publiés ou non, émanant des établissements d'enseignement et de recherche français ou étrangers, des laboratoires publics ou privés. 


\title{
Homogenization of a highly rarefied binary structure of finite diffusivity
}

\author{
I. GRUAIS* \\ Université de Rennes1, I.R.M.A.R, Campus de Beaulieu, \\ 35042 Rennes Cedex, France
}

\begin{abstract}
The homogenization of a binary structure made of very small particles of general shape is performed when the diffusivity is finite and when the size of the particles has a critical value with respect to a rarefaction number. The limit problem involves an auxiliary function which can be interpreted as the solution of a problem of cellular type, thus filling the gap with classical methods of multiple scales.
\end{abstract}

Keywords: Homogenization; Multiple scale method; Diffusion; Binary structure

2000 Mathematics Subject Classifications: 35B27; 35Q72; 74Q05; 74Q15; 76M50

\section{Introduction}

This study follows a collection of three articles [2-4] dealing with the modelization of rarefied binary structures where possibly spherical particles are embedded in an ambiantal medium. The ratio of the radius of the particles and the size of the network is used as main criteria to analyze the limit behavior of the resulting suspension. The case where this ratio is of unity order is well-known and has been classically studied through homogenization methods. Thus, our interest focused on the other cases and more specifically on rarefied suspensions where the particles are very small in comparison with the size of the underlying network. A rarefaction number depending on the radius of the particles was introduced to characterize the limit problem and actually defines what was meant by very small. However, it appeared that when this

*Email: isabelle.gruais@univ-rennesl.fr 
rarefaction number has a critical value, then finite diffusivity is an obstruction to our method. This case is analyzed in the present article using methods that were already presented in a previous work [6].

Although our model suffers from the restrictions due to the choice of the homogenization method, we claim that it is also physically relevant owing to recent studies on microstructures either in optical or porous media.

Indeed, the binary representation of porous media can provide a direct means for the characterization of their internal structure. However, the commonly used concepts of pore, grain [2,3] or fiber [1] models inevitably introduce a significant degree of approximation to the actual structure and rarefied transport prevails in the continuum limit $[3,4]$.

Moreover, optical fields can induce forces between microscopic objects, thus giving rise to new structures of matter. It was demonstrated that optical fields can produce forces on neutral particles and this mechanical action has been used in optical tweezers and more recently in optical force microscopy. The possibility of binding objects through optical forces and thus create microstructures has been pointed out. An application of this principle lies in laser trapped mirrors which may offer an interesting alternative for very large space telescopes. Building laser-trapped sheet structure for use as an astronomical mirror requires investigations on micronscale particles. At the microscopic level, light-matter interactions can organize colloidal matter via a process known as optical binding. Optical binding refers to the creation of arrays of microparticles formed in the presence of laser fields, the inter-particle spacing being determined by the refocusing and/or scattering of the laser fields by the microparticles. As a matter of fact, experimental work on optical binding deals with water suspensions of microspheres. In this respect, the development of nanotechnologies requires studying of transport processes of nanoparticles in fluids. In turn, to control these processes it is necessary to study mechanisms of nanoparticles velocity relaxation in gases and liquids. The key to the solution of these problems is the description of the nanoparticles diffusion in liquids and gases. The size of nanoparticles lies between the molecule and large dispersed particles ones $\left(R \in\left[10^{-7}, 5 \times 10^{-6}\right] \mathrm{sm}\right)$. Therefore, gas nanosuspensions (gas + nanoparticles) and nanosuspensions (liquid + nanoparticles) are essentially different from gas mixtures, ordinary gas suspensions and suspensions.

The present study is based on references [2-4] which were devoted to the mathematical analysis of nanoparticles diffusion in gases and liquids.

It is organized as follows. Section 2 is devoted to the main notations and to the description of the model. In section 3, we show that the analog of Proposition 5.2 of [4] still holds although the localizing operators of [4] are of no use in the case under consideration. Section 4 is devoted to a reformulation of a two-scale convergence result of [6]. The limit problem is determined in section 5 and the main result is stated in Theorem 5.4. The capacitary term is explicited in Proposition 5.5 for the particular case of spherical particles. Theorem 6.1 of section 6 yields an a posteriori justification of the choice of the regularity of external forces. Section 7 makes the link with the two-scale convergence method (see [6] and references therein) and we show in section 8 that the limit problem actually involves an auxiliary problem of cellular type. Finally, in the appendix, we state and prove a maximum principle that was used in Proposition 5.3. 


\section{Rarefied diffusion}

Let $\Omega \subseteq \mathbf{R}^{N}, N \geq 3$, be an open Lipschitz domain and let $D$ be an open set of class $C^{1}$ contained in $B(0,1)$. Let us denote:

$$
Y:=\left(-\frac{1}{2},+\frac{1}{2}\right)^{N}
$$

For some $0<r_{\varepsilon} \ll \varepsilon$, we set

$$
\begin{array}{ll}
Y_{\varepsilon}^{k}:=\varepsilon k+\varepsilon Y, \quad k \in \mathbf{Z}^{N}, & D_{\varepsilon}^{k}:=\varepsilon k+r_{\varepsilon} D, \\
\mathbf{Z}_{\varepsilon}:=\left\{k \in \mathbf{Z}^{N}, Y_{\varepsilon}^{k} \subset \Omega\right\}, & \Omega_{Y_{\varepsilon}}:=\bigcup_{k \in \mathbf{Z}_{\varepsilon}} Y_{\varepsilon}^{k} .
\end{array}
$$

We consider that $\Omega$ is occupied by a mixture of two different materials, one of them forming the ambiantal connected phase, the other being concentrated in a $\varepsilon$-periodical suspension of small particles of general shape. The suspension, denoted by $D_{\varepsilon}$, is defined by the following reunion

$$
D_{\varepsilon}:=\cup_{k \in \mathbf{Z}_{\varepsilon}} D_{\varepsilon}^{k}
$$

and obviously $\left|D_{\varepsilon}\right| \rightarrow 0$ as $\varepsilon \rightarrow 0$. The fluid domain, denoted by $\Omega_{\varepsilon}$, is defined by $\Omega_{\varepsilon}:=\Omega \backslash \bar{D}_{\varepsilon}$.

We consider the problem which governs the diffusion throughout our binary structure. Denoting by $a_{\varepsilon}$ the relative mass density of the concentration, we assume that, although the volume of the suspension is vanishing, its mass is of unity order, namely:

$$
a_{\varepsilon}\left|D_{\varepsilon}\right|=a>0 .
$$

If $b_{\varepsilon}>0$ denotes the relative diffusivity of the suspension, and if we assume, without loss of generality, that $|\Omega|=1$, then we consider the nondimensional from of the problem, namely: To find $u^{\varepsilon}$ solution of

$$
\begin{aligned}
& -b_{\varepsilon} \Delta u^{\varepsilon}+a \frac{u^{\varepsilon}}{\left|D_{\varepsilon}\right|}=f^{\varepsilon} \quad \text { in } D_{\varepsilon} \\
& -\Delta u^{\varepsilon}=f^{\varepsilon} \quad \text { in } \Omega_{\varepsilon} \\
& {\left[u^{\varepsilon}\right]_{\varepsilon}=0 \quad \text { on } \partial D_{\varepsilon}} \\
& \left.b_{\varepsilon} \frac{\left.\partial u^{\varepsilon}\right|_{D_{\varepsilon}}}{\partial n}\right|_{\partial D_{\varepsilon}}=\left.\frac{\left.\partial u^{\varepsilon}\right|_{\Omega_{\varepsilon}}}{\partial n}\right|_{\partial D_{\varepsilon}} \quad \text { on } \partial D_{\varepsilon} \\
& u^{\varepsilon}=0 \text { on } \partial \Omega
\end{aligned}
$$

where $[\cdot]_{\varepsilon}$ is the jump at the interface $\partial D_{\varepsilon}, n$ is the unit normal in the outward direction on $\partial D_{\varepsilon}$, and where $f^{\varepsilon} \in H^{-1}(\Omega)$.

The variational formulation of the problems (6)-(10) reads:

To find $u^{\varepsilon} \in H_{0}^{1}(\Omega)$ satisfying

$$
a \int_{D_{\varepsilon}} u^{\varepsilon} w+b_{\varepsilon} \int_{D_{\varepsilon}} \nabla u^{\varepsilon} \nabla w+\int_{\Omega_{\varepsilon}} \nabla u^{\varepsilon} \nabla w=\left\langle f^{\varepsilon}, w\right\rangle, \quad \forall w \in H_{0}^{1}(\Omega)
$$

where $\langle\cdot, \cdot\rangle$ denotes the duality product between $H^{-1}(\Omega)$ and $H_{0}^{1}(\Omega)$. 
To study the asymptotic behavior of $u^{\varepsilon}$ when $\varepsilon \rightarrow 0$, we make the following additional hypotheses: namely, the relative diffusivity is bounded below by a positive constant:

$$
b_{\varepsilon} \geq b_{0}>0, \quad \forall \varepsilon>0,
$$

and there exists $f \in H^{-1}(\Omega)$ such that

$$
f^{\varepsilon} \rightarrow f \text { in } H^{-1}(\Omega)
$$

Proposition 2.1 Under the assumptions (12)-(13),

$$
u^{\varepsilon} \text { is bounded in } H_{0}^{1}(\Omega) \text {. }
$$

Moreover, there exists $C>0$, independent of $\varepsilon$, such that

$$
f_{D_{\varepsilon}}\left|u^{\varepsilon}\right|^{2} \mathrm{~d} x+b_{\varepsilon}\left|\nabla u^{\varepsilon}\right|_{D_{\varepsilon}}^{2} \leq C
$$

\section{Homogenization of the case $r_{\varepsilon}=\mathcal{O}\left(\varepsilon^{N /(N-2)}\right)$ when the relative diffusivity $b_{\varepsilon}$ is finite}

We assume that $b_{\varepsilon}$ is finite, say $b_{\varepsilon}=a=1$, which leads to the following problem:

To find $u^{\varepsilon} \in H_{0}^{1}(\Omega)$ solution of:

$$
\begin{gathered}
-\Delta u^{\varepsilon}+\frac{u^{\varepsilon}}{\left|D_{\varepsilon}\right|}=f^{\varepsilon} \quad \text { in } D_{\varepsilon} \\
-\Delta u^{\varepsilon}=f^{\varepsilon}, \quad \text { in } \Omega_{\varepsilon} \\
{\left[u^{\varepsilon}\right]_{\partial D_{\varepsilon}}=\left[\frac{\partial u^{\varepsilon}}{\partial n}\right]_{\partial D_{\varepsilon}}=0 .}
\end{gathered}
$$

The variational formulation becomes:

$$
u^{\varepsilon} \in H_{0}^{1}(\Omega) \quad \text { and } \quad \forall \varphi \in H_{0}^{1}(\Omega), \quad \int_{\Omega} \nabla u^{\varepsilon} \nabla \varphi+f_{D_{\varepsilon}} u^{\varepsilon} \varphi=\left\langle f^{\varepsilon}, \varphi\right\rangle
$$

where $\langle\cdot, \cdot\rangle$ denotes the duality product between $H_{0}^{1}(\Omega)$ and $H^{-1}(\Omega)$.

Proposition 3.1 There exists $v \in L^{2}(\Omega)$ such that

$$
\int_{D_{\varepsilon}} u^{\varepsilon} \varphi \mathrm{d} x \rightarrow \int_{\Omega} v \varphi \mathrm{d} x, \quad \forall \varphi \in C_{c}(\Omega) .
$$

Proof Let $M_{\varepsilon}$ denote the operator $M_{\varepsilon}: C_{c}(\Omega) \rightarrow L^{2}(\Omega)$ fined by

$$
M_{\varepsilon}(\varphi)(x):=\sum_{k \in \mathbf{Z}_{\varepsilon}}\left(f_{Y_{\varepsilon}^{k}} \varphi(y) \mathrm{d} y\right) 1_{D_{\varepsilon}^{k}}(x)
$$


Then, the uniform continuity of $\varphi \in C_{c}(\Omega)$ immediately yields:

$$
\lim _{\varepsilon \rightarrow 0} f_{D_{\varepsilon}}\left|\varphi-M_{\varepsilon}(\varphi)\right|^{2} \mathrm{~d} x=0 .
$$

Let $\varphi \in C_{c}(\Omega)$. We have

$$
f_{D_{\varepsilon}} u^{\varepsilon} \varphi \mathrm{d} x=f_{D_{\varepsilon}} u^{\varepsilon}\left(\varphi-M_{\varepsilon}(\varphi)\right) \mathrm{d} x+f_{D_{\varepsilon}} u^{\varepsilon} M_{\varepsilon}(\varphi) \mathrm{d} x
$$

with

$$
\begin{aligned}
\left|f_{D_{\varepsilon}} u^{\varepsilon}\left(\varphi-M_{\varepsilon}(\varphi)\right) \mathrm{d} x\right| & \leq\left(f_{D_{\varepsilon}}\left|u^{\varepsilon}\right|^{2}\right)^{1 / 2}\left(f_{D_{\varepsilon}}\left|\varphi-M_{\varepsilon}(\varphi)\right|^{2} \mathrm{~d} x\right)^{1 / 2} \\
& \leq C\left(f_{D_{\varepsilon}}\left|\varphi-M_{\varepsilon}(\varphi)\right|^{2} \mathrm{~d} x\right)^{1 / 2}
\end{aligned}
$$

where we have taken into account (15). From (21), we deduce that

$$
\lim _{\varepsilon \rightarrow 0} \int_{D_{\varepsilon}} u^{\varepsilon}\left(\varphi-M_{\varepsilon}(\varphi)\right) \mathrm{d} x=0
$$

The remaining term in (22) also reads

$$
f_{D_{\varepsilon}} u^{\varepsilon} M_{\varepsilon}(\varphi) \mathrm{d} x=\int_{\Omega} \varphi P_{\varepsilon}\left(u^{\varepsilon}\right) \mathrm{d} x
$$

where $P_{\varepsilon}$ denotes the operator $L^{2}(\Omega) \rightarrow L^{2}(\Omega)$ defined by

$$
P_{\varepsilon}(\varphi)(x):=\sum_{k \in \mathbf{Z}_{\varepsilon}}\left(f_{D_{\varepsilon}^{k}} \varphi(y) \mathrm{d} y\right) 1_{Y_{\varepsilon}^{k}}(x), \quad \text { a.e. in } \Omega, \quad \forall w \in L^{2}(\Omega) .
$$

Note that

$$
\left|P_{\varepsilon}\left(u^{\varepsilon}\right)\right|_{\Omega}^{2}=\sum_{k \in \mathbf{Z}_{\varepsilon}} \int_{Y_{\varepsilon}^{k}}\left|f_{D_{\varepsilon}^{k}} u^{\varepsilon}\right|^{2} \leq C f_{D_{\varepsilon}}\left|u^{\varepsilon}\right|^{2} \leq C
$$

and thus, at least for some subsequence: there exists $v \in L^{2}(\Omega)$ such that

$$
P_{\varepsilon}\left(u^{\varepsilon}\right) \rightarrow v \text { in } L^{2}(\Omega)
$$

After substitution into (24), this yields

$$
\lim _{\varepsilon \rightarrow 0} \int_{D_{\varepsilon}} u^{\varepsilon} M_{\varepsilon}(\varphi) \mathrm{d} x=\int_{\Omega} v \varphi \mathrm{d} x
$$

We conclude after substitution of (23) and (25) into (22). 


\section{A convergence result}

Definition 4.1 For any $\varphi \in \mathcal{D}(\Omega)$, we set

$$
N_{\varepsilon}(\varphi)=\sum_{k \in \mathbf{Z}_{\varepsilon}} f_{Y_{\varepsilon}^{k}} \varphi(\xi) \mathrm{d} \xi 1_{Y_{\varepsilon}^{k}}(x)=: \sum_{k \in \mathbf{Z}_{\varepsilon}} N_{\varepsilon}^{k}(\varphi) 1_{Y_{\varepsilon}^{k}}(x) .
$$

Definition 4.2 For any $x \in \Omega_{Y_{\varepsilon}}=: \cup_{k \in \mathbf{Z}_{\varepsilon}} Y_{\varepsilon}^{k} \subset \Omega$, we set

$$
y^{\varepsilon}(x)=\frac{1}{r_{\varepsilon}}\left(x-\varepsilon\left[\frac{x}{\varepsilon}\right]\right)
$$

so that

$$
\begin{aligned}
& x \in \Omega_{Y^{\varepsilon}} \Longleftrightarrow y^{\varepsilon} \in K_{\varepsilon}:=\frac{\varepsilon}{r_{\varepsilon}} Y \\
& x \in \Omega_{\varepsilon} \Longleftrightarrow y^{\varepsilon} \in K_{\varepsilon} \backslash D \text { and } x \in D_{\varepsilon} \Longleftrightarrow y^{\varepsilon} \in D .
\end{aligned}
$$

Proposition 4.3 Let $\phi \in C_{c}\left(\mathbf{R}^{N}\right)$ and $\psi \in C(\Omega)$. Then,

$$
\int_{\Omega} \phi\left(y^{\varepsilon}\right) N_{\varepsilon}(\psi)=\int_{\Omega} \psi(x)\left(f_{K_{\varepsilon}} \phi\right) .
$$

In particular,

$$
f_{D_{\varepsilon}} \phi\left(y^{\varepsilon}\right) N_{\varepsilon}(\psi)=\frac{1}{\varepsilon^{N} \operatorname{card}_{\varepsilon}} \int_{\Omega} \psi(x)\left(f_{D} \phi\right) \sim \int_{\Omega} \psi(x)\left(f_{D} \phi\right) .
$$

Proof We have

$$
\begin{aligned}
\int_{\Omega} \phi\left(y^{\varepsilon}\right) N_{\varepsilon}(\psi) & =\sum_{k \in \mathbf{Z}_{\varepsilon}} \int_{Y_{\varepsilon}^{k}} \phi\left(y^{\varepsilon}\right) N_{\varepsilon}^{k}(\psi)=r_{\varepsilon}^{N} \sum_{k \in \mathbf{Z}_{\varepsilon}} \int_{K_{\varepsilon}} \phi(y) N_{\varepsilon}^{k}(\psi) \\
& =\frac{r_{\varepsilon}^{N}}{\varepsilon^{N}} \sum_{k \in \mathbf{Z}_{\varepsilon}} \int_{Y_{\varepsilon}^{k}} \psi(x) \int_{K_{\varepsilon}} \phi(y)=f_{\Omega \times K_{\varepsilon}} \psi(x) \phi(y),
\end{aligned}
$$

which yields (26). There results

$$
f_{D_{\varepsilon}} \phi\left(y^{\varepsilon}\right) N_{\varepsilon}(\psi)=\frac{1}{\left|K_{\varepsilon}\right|\left|D_{\varepsilon}\right|} \int_{\Omega} \psi(x)\left(\int_{D} \phi\right)
$$

where $\left|K_{\varepsilon}\right|=\left(\varepsilon / r_{\varepsilon}\right)^{N}$. Note that

$$
\left|D_{\varepsilon}\right|=\operatorname{card} \mathbf{Z}_{\varepsilon} r_{\varepsilon}^{N}|D|=\varepsilon^{N} \operatorname{card} \mathbf{Z}_{\varepsilon}\left(\frac{r_{\varepsilon}}{\varepsilon}\right)^{N}|D|=\varepsilon^{N} \operatorname{card} \mathbf{Z}_{\varepsilon} \frac{|D|}{\left|K_{\varepsilon}\right|}
$$

and therefore $\left(1 /\left|K_{\varepsilon}\right|\right)=\left(1 / \varepsilon^{N} \operatorname{card}_{\varepsilon}\right)\left(\left|D_{\varepsilon}\right| /|D|\right)$, which yields (27). 


\section{The limit problem}

Let $\chi \lambda \in H_{0}^{1}\left(B_{\lambda}\right)$ be the solution of

$$
\begin{gathered}
-\gamma \Delta_{y} \chi \lambda+\frac{\chi \lambda}{|D|}=\frac{1}{|D|}, \quad \text { in } D \\
-\gamma \Delta_{y} \chi \lambda=0, \quad \text { in } B_{\lambda} \backslash D \\
{[\chi \lambda]_{\partial D}=\left[\frac{\partial \chi \lambda}{\partial n}\right]_{\partial D}=0} \\
\chi \lambda=0, \quad \text { on } \partial B_{\lambda} .
\end{gathered}
$$

Definition 5.1 Let $\mathcal{R}$ a set of control sequences defined by:

$$
\mathcal{R}=\left\{\left(R_{\varepsilon}\right)_{\varepsilon}, r_{\varepsilon} \ll R_{\varepsilon} \ll \varepsilon\right\} .
$$

For every sequence $\left(R_{\varepsilon}\right)_{\varepsilon} \in \mathcal{R}$, we introduce the associated control zone, namely:

$$
\mathcal{C}_{\varepsilon}=\underset{k \in \mathbf{Z}_{k}}{\cup}\left(B_{\varepsilon}^{k} \backslash \bar{D}_{\varepsilon}^{k}\right)
$$

where the $B_{\varepsilon}^{k}=B\left(\varepsilon k, R_{\varepsilon}\right), k \in \mathbf{Z}_{\varepsilon}$, denote balls centered at the nodes of the network.

Letting $\lambda_{\varepsilon}=r_{\varepsilon}^{-1} R_{\varepsilon}$, we establish:

Proposition 5.2 Let $\chi^{\varepsilon}$ be defined in $\Omega_{Y_{\varepsilon}}$ by $\chi^{\varepsilon}=\chi \lambda_{\varepsilon}\left(y^{\varepsilon}\right)$. Then, $\chi^{\varepsilon}$ is solution of

$$
\begin{gathered}
-\Delta \chi^{\varepsilon}+\frac{\chi^{\varepsilon}}{\left|D^{\varepsilon}\right|}=\mu_{\varepsilon}, \quad \text { in } D_{\varepsilon} \\
-\Delta \chi^{\varepsilon}=0, \quad \text { in } C_{\varepsilon} \\
{\left[\chi^{\varepsilon}\right]_{\partial D_{\varepsilon}}=\left[\frac{\partial \chi^{\varepsilon}}{\partial n}\right]_{\partial D_{\varepsilon}}=0} \\
\chi^{\varepsilon}=0 \quad \text { in } \Omega_{\varepsilon} \backslash C_{\varepsilon} .
\end{gathered}
$$

where $\mu_{\varepsilon} \sim\left(1 /\left|D_{\varepsilon}\right|\right)$. Moreover, $\chi^{\varepsilon} \rightarrow 0$ in $L^{2}(\Omega)$ and

$$
\left|\nabla \chi^{\varepsilon}\right|_{\Omega}^{2}=\gamma_{\varepsilon} \int_{\Omega \times B_{\lambda_{\varepsilon}}}\left|\nabla_{y} \chi \lambda_{\varepsilon}\right|^{2} \leq C .
$$

Proof An immediate computation yields formulas (34)-(37). Moreover,

$$
\begin{aligned}
\left|\chi^{\varepsilon}\right|_{\Omega}^{2} & =f_{\Omega \times K_{\varepsilon}}\left|\chi \lambda_{\varepsilon}\right|^{2}=\left(\frac{r_{\varepsilon}}{\varepsilon}\right)^{N} \int_{\Omega \times B_{\lambda_{\varepsilon}}}\left|\chi \lambda_{\varepsilon}\right|^{2} \sim\left(\frac{r_{\varepsilon}}{\varepsilon}\right)^{N} \int_{\Omega \times \mathbf{R}^{N}}\left|\chi^{0}\right|^{2} \rightarrow 0 . \\
\left|\nabla \chi^{\varepsilon}\right|_{\Omega}^{2} & =\gamma_{\varepsilon} \int_{\Omega \times K_{\varepsilon}}\left|\nabla_{y} \chi \lambda_{\varepsilon}\right|^{2} \sim \gamma \int_{\Omega \times \mathbf{R}^{N}}\left|\nabla_{y} \chi^{0}\right|^{2}<+\infty
\end{aligned}
$$


where $\chi^{0}$ is the unique solution of

$$
\begin{gathered}
-\gamma \Delta_{y} \chi^{0}+\frac{\chi^{0}}{|D|}=\frac{1}{|D|}, \quad \text { in } D \\
-\gamma \Delta_{y} \chi^{0}=0 \text { in } \mathbf{R}^{N} \backslash D \\
{\left[\chi^{0}\right]_{\partial D}=\left[\frac{\partial \chi^{0}}{\partial n}\right]_{\partial D}=0}
\end{gathered}
$$

Having in mind the application of a maximum principle for which we refer to Proposition A.1 of the appendix, we need the following:

Proposition 5.3 The solution $\chi^{0}$ of (38)-(40) satisfies the following estimates:

$$
0<1-f_{D} \chi^{0}<1
$$

Proof Multiplying both sides of (38)-(40) by $\chi^{0}$ and applying Green's formula, we get

$$
f_{D} \chi^{0}=f_{D}\left|\chi^{0}\right|^{2}+\gamma \int_{\mathbf{R}^{N} \backslash D}\left|\nabla_{y} \chi^{0}\right|^{2}>0 .
$$

The maximum principle (see Proposition A.1 below) yields, for every $\lambda \geq 1$ :

$$
0=\min \left(\min _{\partial B_{\lambda}} \chi \lambda, 0=f_{\mid B_{\lambda} \backslash D}\right) \leq \chi \lambda \leq \max \left(\max _{\partial B_{\lambda}} \chi \lambda, 1=f_{\mid D}\right)=1 .
$$

In particular,

$$
0 \leq \int_{D} \chi^{0} \varphi \leq \int_{D} \varphi, \quad \forall \varphi \in C(D) \text { such that } \varphi \geq 0 .
$$

Passing to the limit as $\lambda \rightarrow+\infty$, we get

$$
0 \leq f_{D} \chi^{0} \varphi \leq \int_{D} \varphi, \quad \forall \varphi \in C(D) \text { such that } \varphi \geq 0
$$

that is,

$$
0 \leq \chi^{0} \leq 1 \quad \text { in } D
$$

To conclude, we compare $\chi \lambda$ with the solution $w_{\lambda}$ of the following problem

$$
\begin{aligned}
-\Delta w_{\lambda}=0 & \text { in } C_{\lambda}:=B(0, \lambda) \backslash D, \\
w_{\lambda}=1 & \text { on } \partial D, \\
w_{\lambda}=0 & \text { on } S_{\lambda}:=\partial B(0, \lambda) .
\end{aligned}
$$


Classical results in potential theory ensure the convergence of

$$
\operatorname{cap}_{\lambda}(D):=\int_{C_{\lambda}}\left|\nabla w_{\lambda}\right|^{2}
$$

towards the capacity of $D$, namely

$$
\lim _{\lambda \rightarrow+\infty} \operatorname{cap}_{\lambda}(D)=\operatorname{cap}(D):=\int_{\mathbf{R}^{N} \backslash D}\left|\nabla w_{\infty}\right|^{2},
$$

where $w_{\infty}$ is the solution of the exterior Dirichlet problem with respect to $D$. Assume for a while that $f_{D} \chi^{0}=1$. Then, we get that $\chi^{0} \equiv 1$ a.e. in $D$, and thus $\chi^{0}=w_{\infty}$ a.e. in $\mathbf{R}^{N}$, which contradicts

$$
\left[\frac{\partial w_{\infty}}{\partial n}\right]_{\partial D}=-\left.\frac{\partial w_{\infty}}{\partial n}\right|_{\partial\left(\mathbf{R}^{N} \backslash D\right)} \neq 0
$$

where $n$ is the unit outward normal on $\partial\left(\mathbf{R}^{N} \backslash D\right)$, i.e. the unit inward normal on $\partial D$. This leads to a contradiction, and thus $f_{D} \chi^{0} \neq 1$, which achieves the proof.

THEOREM 5.4 Assume that there exists $h \in L^{2}(\Omega)$ such that

$$
\forall \psi \in D(\Omega), \quad f_{\Omega} f^{\varepsilon} \chi^{\varepsilon} N_{\varepsilon}(\psi) \rightarrow \int_{\Omega} h \psi .
$$

Then

$$
u=\frac{h}{1-f_{D} \chi^{0}}
$$

Proof We have

$$
\chi^{\varepsilon} \rightarrow 0 \text { in } L^{2}(\Omega) \text { strongly. }
$$

The uniform continuity of $\psi$ on $\Omega$ yields:

$$
N_{\varepsilon}(\psi) \rightarrow \quad \text { in } L^{\infty}(\Omega)
$$

Then we get, by definition of $v$ :

$$
f_{D_{\varepsilon}} u^{\varepsilon}\left(1-\chi^{\varepsilon}\right) N_{\varepsilon}(\psi) \rightarrow \int_{\Omega} v \psi
$$

Moreover

$$
f_{D_{\varepsilon}} u^{\varepsilon}\left(1-\chi^{\varepsilon}\right) N_{\varepsilon}(\psi)=\int_{\Omega} u^{\varepsilon} \frac{1_{D_{\varepsilon}}}{\left|D_{\varepsilon}\right|}\left(1-\chi^{\varepsilon}\right) N_{\varepsilon}(\psi)
$$


where

$$
\begin{aligned}
\int_{\Omega} \frac{1_{D_{\varepsilon}}}{\left|D_{\varepsilon}\right|}\left(1-\chi^{\varepsilon}\right) N_{\varepsilon}(\psi) & =f_{D_{\varepsilon}}\left(1-\chi^{\varepsilon}\right) N_{\varepsilon}(\psi)=\frac{1}{\varepsilon^{N} \operatorname{card} \mathbf{Z}_{\varepsilon}} f_{\Omega \times D}\left(1-\chi \lambda_{\varepsilon}(y)\right) \psi(x) \\
& =\frac{1}{\varepsilon^{N} \operatorname{card}_{\varepsilon}} \int_{\Omega} \psi(x)\left(f_{\Omega}\left(1-\chi_{\lambda_{\varepsilon}}(y)\right)\right) \rightarrow \int_{\Omega}\left(f_{D}\left(1-\chi^{0}\right)\right)
\end{aligned}
$$

and thus

$$
\frac{1_{D_{\varepsilon}}}{\left|D_{\varepsilon}\right|}\left(1-\chi^{\varepsilon}\right) \rightarrow f_{D}\left(1-\chi^{0}\right)=1-f_{D} \chi^{0} \quad \text { in } L^{2}(\Omega) \text { weakly. }
$$

As $u^{\varepsilon} \rightarrow u$ in $L^{2}(\Omega)$ strongly and taking into account (46), we infer that

$$
f_{D_{\varepsilon}} u^{\varepsilon}\left(1-\chi^{\varepsilon}\right) N_{\varepsilon}(\psi) \rightarrow \int_{\Omega} u\left(1-f_{D} \chi^{0}\right) \psi
$$

Comparing with (47), we conclude that

$$
v=u\left(1-f_{D} \chi^{0}\right)
$$

Moreover, arguing as in Tartar's energy method, we compute

$$
\begin{gathered}
\int_{\Omega} \nabla u^{\varepsilon} \nabla \chi^{\varepsilon} N_{\varepsilon}(\psi)+f_{D_{\varepsilon}} u^{\varepsilon} \chi^{\varepsilon} N_{\varepsilon}(\psi)=\int_{\Omega} f^{\varepsilon} \chi^{\varepsilon} N_{\varepsilon}(\psi) \\
\int_{\Omega} \nabla u^{\varepsilon} \nabla \chi^{\varepsilon} N_{\varepsilon}(\psi)+f_{D_{\varepsilon}} u^{\varepsilon} \chi^{\varepsilon} N_{\varepsilon}(\psi)=f_{\Omega} u^{\varepsilon} N_{\varepsilon}(\psi) .
\end{gathered}
$$

Substracting (50) from (49), we get

$$
f_{D_{\varepsilon}} u^{\varepsilon} N_{\varepsilon}(\psi)=\int_{\Omega} f^{\varepsilon} \chi^{\varepsilon} N_{\varepsilon}(\psi)
$$

Then, passing to the limit as $\varepsilon \rightarrow 0$ in (51) and taking advantage of the hypothesis (44), we deduce that

$$
\int_{\Omega} v \psi=\int_{\Omega} h \psi
$$

that is, $v=h$. The result follows from (48).

Remark 1 The quantity $1-f_{D} \chi^{0}$ is related to the mean capacity of $\chi^{0}$ on the open set $D$ through the identity:

$$
1-\int_{D} \chi^{0}=\gamma \int_{\partial D} \chi^{0}\left(-\frac{\partial w_{\infty}}{\partial n}\right)=\gamma \int_{\partial D}\left(-\frac{\partial \chi^{0}}{\partial n}\right)
$$


Indeed, the same arguments as above with $w_{\infty}$ as test function lead to

$$
1=f_{D} \chi^{0}+\gamma \int_{\mathbf{R}^{N} \backslash D} \nabla_{y} \chi^{0} \nabla_{y} w_{\infty}
$$

where

$$
\gamma \int_{\mathbf{R}^{N} \backslash D} \nabla_{y} \chi^{0} \nabla_{y} w_{\infty}=\gamma \int_{\partial D} \chi^{0}\left(-\frac{\partial w_{\infty}}{\partial n}\right)
$$

and

$$
\gamma \int_{\mathbf{R}^{N} \backslash D} \nabla_{y} \chi^{0} \nabla_{y} w_{\infty}=\gamma \int_{\partial D}\left(-\frac{\partial \chi^{0}}{\partial n}\right)
$$

which yields the result.

Proposition 5.5 In the particular case where $D=B(0,1)$ is the unit ball of $\mathbf{R}^{3}$, we have

$$
\left.f_{D} \chi^{0}=\frac{3(\omega-\tanh \omega)}{\omega^{3}} \in\right] 0,1\left[, \quad \text { where } \omega:=\sqrt{\frac{3}{4 \pi \gamma}} .\right.
$$

Proof For every $\lambda>1, \chi \lambda$ has the spherical symmetry and thus $\chi \lambda=\chi \lambda(r)$ is a radial function. There results that $\chi \lambda$ solves

$$
\begin{gathered}
-\gamma \frac{\mathrm{d}}{\mathrm{d} r}\left(r^{2} \frac{\mathrm{d}}{\mathrm{d} r} \chi \lambda\right)+\frac{3}{4 \pi} r^{2} \chi \lambda=\frac{3}{4 \pi} r^{2}, \quad 0<r<1 \\
-\gamma \frac{\mathrm{d}}{\mathrm{d} r}\left(r^{2} \frac{\mathrm{d}}{\mathrm{d} r} \chi \lambda\right)=0, \quad 1<r<\lambda \\
\chi \lambda\left(1^{+}\right)=\chi\left(1^{-}\right), \quad \frac{\mathrm{d}}{\mathrm{d} r} \chi \lambda\left(1^{+}\right)=\frac{\mathrm{d}}{\mathrm{d} r} \chi \lambda\left(1^{-}\right) \\
\chi \lambda(\lambda)=1 .
\end{gathered}
$$

Setting $\psi=r(\chi \lambda-1)$ for $0 \leq r \leq 1$ we find that solves the following differential equation

$$
-\gamma \frac{\mathrm{d}^{2}}{\mathrm{~d} r^{2}}+\frac{3}{4 \pi}=0, \quad 0<r<1
$$

whose general solution takes the form

$$
=A e^{\omega r}+B e^{-\omega r} .
$$

There follows

$$
\chi \lambda=1+A \frac{e^{\omega r}}{r}+B \frac{e^{-\omega r}}{r}, \text { for } 0<r<1
$$


where the limit

$$
\chi \lambda \sim 1+\frac{A+B}{r} \quad \text { as } r \rightarrow 0
$$

is defined as soon as $A+B=0$, which leads to

$$
\chi \lambda=1+2 A \frac{\sinh (\omega r)}{r} \quad 0<r<1 \text { and } \chi \lambda(0)=1+2 A \omega
$$

For every $1<r<\lambda$, direct computation as well as the boundary condition (53) yield

$$
\chi \lambda(r)=C\left(\frac{1}{\lambda}-\frac{1}{r}\right)
$$

where the constant $C$ remains to be determined. Note that (52) leads to:

$$
C=2 A(\omega \cosh \omega-\sinh \omega)
$$

and

$$
2 A=\frac{\lambda}{(1-\lambda) \omega \cosh \omega-\sinh \omega},
$$

successively. Finally, we get

$$
\begin{aligned}
& \chi \lambda(r)=1+\frac{\lambda}{(1-\lambda) \omega \cosh \omega-\sinh \omega} \frac{\sinh (\omega r)}{r}, \quad \text { if } 0<r<1 \\
& \chi \lambda(r)=\frac{(r-\lambda)}{r} \frac{(\omega \cosh \omega-\sinh \omega)}{(1-\lambda) \omega \cosh \omega-\sinh \omega}, \quad \text { if } 1<r<\lambda .
\end{aligned}
$$

Then, a direct computation shows that

$$
1-f_{D} \chi \lambda=-\frac{3 \lambda}{\omega^{2}}\left(\frac{\omega \cosh \omega-\sinh \omega}{(1-\lambda) \omega \cosh \omega-\sinh \omega}\right)
$$

As $\lambda \rightarrow+\infty$ :

$$
1-f_{D} \chi^{0}=\frac{3}{\omega^{3}}(\omega-\tanh \omega)
$$

and considering the variations of $\omega \mapsto\left(3 / \omega^{3}\right)(\omega-\tanh \omega)$ we get $0<f_{D} \chi^{0}<1$. 


\section{An alternative result}

If we assume that

$$
f^{\varepsilon} \rightarrow f \text { in } L^{2}(\Omega)
$$

then the limit problem is much simpler (see Theorem 6.1 below).

In that case, the variational formulation reads

$$
u^{\varepsilon} \in H_{0}^{1}(\Omega) \quad \text { and } \quad \forall \varphi \in H_{0}^{1}(\Omega), \quad \int_{\Omega} \nabla u^{\varepsilon} \nabla \varphi+\int_{D_{\varepsilon}} u^{\varepsilon} \varphi=\int_{\Omega} f^{\varepsilon} \varphi
$$

Setting $\varphi=u^{\varepsilon}$ in (55), we find that the energy is bounded, which yields the a priori estimation:

$$
\left|\nabla u^{\varepsilon}\right|_{\Omega}^{2}+f_{D_{\varepsilon}}\left|u^{\varepsilon}\right|^{2} \leq C
$$

for some generic constant $C>0$ independent of $\varepsilon$.

Passing to the limit as $\varepsilon \rightarrow 0$ and taking into account the above results, we find that

$$
u \in H_{0}^{1}(\Omega) \quad \text { and } \quad \forall \varphi \in H_{0}^{1}(\Omega), \quad \int_{\Omega} \nabla u \nabla \varphi+\int_{\Omega} v \varphi=\int_{\Omega} f \varphi
$$

THEOREM 6.1 The limit $u$ is solution of

$$
-\Delta u=f \quad \text { in } \Omega, \quad u \in H_{0}^{1}(\Omega)
$$

Proof Let $\psi \in C_{c}^{1}(\Omega)$. Taking $\Phi^{\varepsilon}=\chi^{\varepsilon} N_{\varepsilon}(\psi)$ as a test function in the variational formulation (55), and $u^{\varepsilon} N_{\varepsilon}(\psi)$ as a test function in the variational formulation of (34)-(37), we successively find:

$$
\begin{aligned}
& \int_{\Omega} \nabla u^{\varepsilon} \nabla \chi^{\varepsilon} N_{\varepsilon}(\psi)+\int_{D_{\varepsilon}} u^{\varepsilon} \chi^{\varepsilon} N_{\varepsilon}(\psi)=\int_{\Omega_{\varepsilon}} f^{\varepsilon} \chi^{\varepsilon} N_{\varepsilon}(\psi) \\
& \int_{\Omega} \nabla u^{\varepsilon} \nabla \chi^{\varepsilon} N_{\varepsilon}(\psi)+\int_{D_{\varepsilon}} u^{\varepsilon} \chi^{\varepsilon} N_{\varepsilon}(\psi)=\int_{D_{\varepsilon}} u^{\varepsilon} N_{\varepsilon}(\psi) .
\end{aligned}
$$

Comparing (57) and (58), we deduce that

$$
\int_{D_{\varepsilon}} u^{\varepsilon} N_{\varepsilon}(\psi)=\int_{\Omega_{\varepsilon}} f^{\varepsilon} \chi^{\varepsilon} N_{\varepsilon}(\psi)
$$

with

$$
f_{D_{\varepsilon}} u^{\varepsilon} N_{\varepsilon}(\psi) \rightarrow \int_{\Omega} v \psi, \quad \int_{\Omega_{\varepsilon}} f^{\varepsilon} \chi^{\varepsilon} N_{\varepsilon}(\psi) \rightarrow 0
$$


that is, $f_{\Omega} v \psi=0$. There results $v=0$. To conclude, note that, taking into account (56), we find that $u$ solves

$$
-\Delta u+v=f, \quad \text { in } \Omega
$$

which achieves the proof.

\section{The limit is necessary: justification by the two-scale convergence [6]}

Using the two-scale convergence method, we find that $\chi^{0}$ defined as the solution of (38)-(40) is a natural choice. To show this, the convergence defined in [6] is revisited relying on notations $[3,4]$.

Definition 7.1 For every $\theta \in H_{0}^{1}(\Omega)$, define $\tilde{\theta}^{\varepsilon} \in L^{2}\left(\Omega \times \mathbf{R}^{N}\right)$ as

$$
\tilde{\theta}^{\varepsilon}(x, y)=\sum_{k \in \mathbf{Z}_{\varepsilon}} \theta\left(\varepsilon k+r_{\varepsilon} y\right) 1_{Y_{\varepsilon}^{k}}(x) .
$$

Proposition 7.2 Let $\phi \in \mathcal{D}\left(\mathbf{R}^{N}\right)$ and $\psi \in D(\Omega)$. Then

$$
\int_{\Omega} u^{\varepsilon} \phi\left(y^{\varepsilon}\right) N_{\varepsilon}(\psi)=\int_{\Omega} \psi(x)\left(f_{K_{\varepsilon}} \tilde{u}^{\varepsilon} \phi\right) .
$$

Proof We have

$$
\begin{aligned}
\int_{\Omega} u^{\varepsilon} \phi\left(y^{\varepsilon}\right) N_{\varepsilon}(\psi) & =\sum_{k \in \mathbf{Z}_{\varepsilon}} \int_{Y_{\varepsilon}^{k}} u^{\varepsilon} \phi\left(y^{\varepsilon}\right) N_{\varepsilon}^{k}(\psi)=r_{\varepsilon}^{N} \sum_{k \in \mathbf{Z}_{\varepsilon}} \int_{K_{\varepsilon}} u^{\varepsilon}\left(\varepsilon k+r_{\varepsilon} y\right) \phi(y) N_{\varepsilon}^{k}(\psi) \\
& =\frac{r_{\varepsilon}^{N}}{\varepsilon^{N}} \sum_{k \in \mathbf{Z}_{\varepsilon}} \int_{Y_{\varepsilon}^{k}} \psi(x) \int_{K_{\varepsilon}} u^{\varepsilon}\left(\varepsilon k+r_{\varepsilon} y\right) \phi(y)=\int_{\Omega \times K_{\varepsilon}} \psi(x) \tilde{u}^{\varepsilon}(x, y) \phi(y) .
\end{aligned}
$$

COROllary 7.3 For every $\theta, \eta \in L^{2}(\Omega), \psi \in C^{0}(\Omega)$, there holds

$$
\int_{\Omega} \theta_{\eta} N_{\varepsilon}(\psi)=\int_{\Omega}\left(f_{K_{\varepsilon}} \tilde{\theta}^{\varepsilon} \tilde{\eta}^{\varepsilon}\right)
$$

As a direct consequence of Corollary 7.3, we have

Proposition 7.4 For every $\theta \in L^{2}(\Omega)$ and every $\psi \in C^{0}(\Omega)$, the following holds true:

$$
\int_{\Omega}|\theta|^{2} N_{\varepsilon}(\psi)=\int_{\Omega} \psi f_{K_{\varepsilon}}\left|\tilde{\theta}^{\varepsilon}\right|^{2}, \quad f_{D_{\varepsilon}}|\varphi|^{2} N_{\varepsilon}(\psi)=\frac{1}{\varepsilon^{N} \operatorname{card}_{\varepsilon}} \int_{\Omega} \psi f_{D}\left|\tilde{\theta}^{\varepsilon}\right|^{2} .
$$


Moreover, if $\theta \in H^{1}(\Omega)$, then

$$
\int_{\Omega}|\nabla \theta|^{2} N_{\varepsilon}(\psi)=\gamma_{\varepsilon} \int_{\Omega}\left|\nabla_{y} \tilde{\theta}^{\varepsilon}\right|_{K_{\varepsilon}}^{2}
$$

Proof Inequalities (60) follow directly from Proposition 7.2. As for (61), note that for every $\theta \in C^{1}(\Omega)$ :

$$
\widetilde{\nabla \theta^{\varepsilon}}(x, y)=\sum_{k \in \mathbf{Z}_{\varepsilon}} \nabla \theta\left(\varepsilon k+r_{\varepsilon} y\right) 1_{Y_{\varepsilon}^{k}}(x)
$$

while

$$
\nabla \tilde{\theta}^{\varepsilon}(x, y)=\nabla_{y} \tilde{\theta}^{\varepsilon}=r_{\varepsilon} \sum_{k \in \mathbf{Z}_{\varepsilon}} \nabla \theta\left(\varepsilon k+r_{\varepsilon} y\right) 1_{Y_{\varepsilon}^{k}}(x)=r_{\varepsilon} \widetilde{\nabla \theta^{\varepsilon}}(x, y) .
$$

There results:

$$
\int_{\Omega}|\nabla \theta|^{2} N_{\varepsilon}(\psi)=\frac{1}{r_{\varepsilon}^{2}} \frac{r_{\varepsilon}^{N}}{\varepsilon^{N}} \int_{\Omega}\left|\nabla_{y} \tilde{\theta}^{\varepsilon}\right|_{K_{\varepsilon}}^{2}=\gamma_{\varepsilon} \int_{\Omega}\left|\nabla_{y} \tilde{\theta}^{\varepsilon}\right|_{K_{\varepsilon}}^{2}
$$

Proposition 7.5 If $\left.\gamma_{\varepsilon} \rightarrow \gamma \in\right] 0,+\infty\left[\right.$, then, the following estimates hold true for $\tilde{u}^{\varepsilon}$ :

$$
\left|\tilde{u}^{\varepsilon}\right|_{\Omega \times D}^{2} \leq C, \quad \int_{\Omega \times K_{\varepsilon}}\left|\nabla_{y} \tilde{u}^{\varepsilon}\right|^{2} \leq C .
$$

Proof We have:

$$
\begin{aligned}
\gamma_{\varepsilon} \int_{\Omega \times K_{\varepsilon}}\left|\nabla_{y} \tilde{u}^{\varepsilon}\right|^{2} & =\int_{\Omega}\left|\nabla u^{\varepsilon}\right|^{2} N_{\varepsilon}(1)=\int_{\Omega_{Y_{\varepsilon}}}\left|\nabla u^{\varepsilon}\right|^{2} \leq\left|\nabla u^{\varepsilon}\right|_{\Omega}^{2} \leq C, \\
f_{\Omega \times D}\left|\tilde{u}^{\varepsilon}\right|^{2} & =\varepsilon^{N} \operatorname{card} \mathbf{Z}_{\varepsilon} \int_{D_{\varepsilon}}\left|u^{\varepsilon}\right|^{2} N_{\varepsilon}(1) \\
& =\frac{\varepsilon^{N} \operatorname{card} \mathbf{Z}_{\varepsilon}}{\left|D_{\varepsilon}\right|} \int_{D_{\varepsilon} \cap \Omega_{Y_{\varepsilon}}}\left|u^{\varepsilon}\right|^{2} \\
& \leq C f_{D_{\varepsilon}}\left|u^{\varepsilon}\right|^{2} \leq C .
\end{aligned}
$$

Proposition 7.6 The following estimate holds true:

$$
\int_{\Omega}\left(\int_{K_{\varepsilon}}\left|\tilde{u}^{\varepsilon}-N_{\varepsilon}\left(u^{\varepsilon}\right)\right|^{2 N /(N-2)}\right)^{(N-2) / N} \leq C .
$$

Proof We have

$$
\int_{\Omega}\left(\int_{K_{\varepsilon}}\left|\tilde{u}^{\varepsilon}-N_{\varepsilon}\left(u^{\varepsilon}\right)\right|^{2 N /(N-2)}\right)^{(N-2) / N}=\int_{\Omega}\left|K_{\varepsilon}\right|^{(N-2) / N}\left(\int_{Y}\left|\tilde{u}^{\varepsilon, \#}-N_{\varepsilon}\left(u^{\varepsilon}\right)\right|^{2 N /(N-2)}\right)^{(N-2) / N}
$$


where we have set

$$
\tilde{u}^{\varepsilon, \#}(\cdot, z):=\tilde{u}^{\varepsilon}\left(\cdot, \frac{\varepsilon}{r_{\varepsilon}} z\right)
$$

Poincaré-Wirtinger's inequality yields:

$$
\begin{gathered}
\int_{\Omega}\left(\int_{K_{\varepsilon}}\left|\tilde{u}^{\varepsilon}-N_{\varepsilon}\left(u^{\varepsilon}\right)\right|^{2 N /(N-2)}\right)^{(N-2) / N} \leq \int_{\Omega}\left|K_{\varepsilon}\right|^{(N-2) / N}\left(\int_{Y} \mid \nabla_{z} \tilde{u}^{\varepsilon,\left.\#\right|^{2}}\right) \\
=\int_{\Omega}\left|K_{\varepsilon}\right|^{(N-2) / N}\left(\int_{K_{\varepsilon}}\left|K_{\varepsilon}\right|^{-1}\left|\nabla_{y} \tilde{u}^{\varepsilon}\right|^{2}\left|K_{\varepsilon}\right|^{2 / N}\right)=\int_{\Omega \times K_{\varepsilon}}\left|\nabla_{y} \tilde{u}^{\varepsilon}\right|^{2} \leq C .
\end{gathered}
$$

Proposition 7.7 For every $\psi \in C^{0}(\Omega)$, there holds

$$
\int_{\Omega} N_{\varepsilon}\left(u^{\varepsilon}\right) \psi \rightarrow \int_{\Omega} u \psi \quad \text { as } \varepsilon \rightarrow 0
$$

Proof We have

$$
\int_{\Omega} N_{\varepsilon}\left(u^{\varepsilon}\right) \psi=\sum_{k \in \mathbf{Z}_{\varepsilon}} \int_{Y_{\varepsilon}^{k}}\left(f_{Y_{\varepsilon}^{k}} u^{\varepsilon}\right)=\sum_{k \in \mathbf{Z}_{\varepsilon}}\left(f_{Y_{\varepsilon}^{k}}\right) \int_{Y_{\varepsilon}^{k}} u^{\varepsilon}=\int_{\Omega} u^{\varepsilon} N_{\varepsilon}(\psi) \rightarrow \int_{\Omega} u \psi
$$

Proposition 7.8 There exists $u^{0}: \Omega \times \mathbf{R}^{N} \rightarrow \mathbf{R}$ that satisfies

$$
u^{0}-u \in L^{2}\left(\Omega ; H^{1}\left(\mathbf{R}^{N}\right)\right) \cap L^{2}\left(\Omega ; L^{2 N /(N-2)}\left(\mathbf{R}^{N}\right)\right)
$$

and, at least for some subsequence,

$$
\tilde{u}^{\varepsilon} \rightarrow u^{0} \quad \text { in } L^{2}\left(\Omega ; H_{\mathrm{loc}}^{1}\left(\mathbf{R}^{N}\right)\right) \cap L^{2}\left(\Omega ; L_{\mathrm{loc}}^{2 N /(N-2)}\left(\mathbf{R}^{N}\right)\right) .
$$

Proof The estimates (62) and (63) also read:

$$
\left|\tilde{u}^{\varepsilon}\right|_{\Omega \times D}^{2} \leq C, \quad \int_{\Omega \times \mathbf{R}^{N}}\left|\nabla_{y} \tilde{u}^{\varepsilon}\right|^{2} \leq C
$$

and

$$
\int_{\Omega}\left(\int_{\mathbf{R}^{N}}\left|\tilde{u}^{\varepsilon}-N_{\varepsilon}\left(u^{\varepsilon}\right) 1_{K_{\varepsilon}}\right|^{2 N /(N-2)}\right)^{(N-2) / N} \leq C .
$$

We deduce the existence of some $u^{0 \#} \in L^{2}\left(\Omega ; H^{1}\left(\mathbf{R}^{N}\right)\right) \cap L^{2}\left(\Omega ; L^{2 N /(N-2)}\left(\mathbf{R}^{N}\right)\right)$ such that, at least for some subsequence:

$$
\tilde{u}^{\varepsilon}-N_{\varepsilon}\left(u^{\varepsilon}\right) 1_{K_{\varepsilon}} \rightarrow u^{0 \#} \quad \text { in } L^{2}\left(\Omega ; H^{1}\left(\mathbf{R}^{N}\right)\right) \cap L^{2}\left(\Omega ; L^{2 N /(N-2)}\left(\mathbf{R}^{N}\right)\right) .
$$


Moreover, (64) implies that

$$
N_{\varepsilon}\left(u^{\varepsilon}\right) 1_{K_{\varepsilon}} \rightarrow u \text { in } L^{2}(\Omega) .
$$

As $\psi \equiv 1 \in H_{\mathrm{loc}}^{1}\left(\mathbf{R}^{N}\right) \cap L_{\mathrm{loc}}^{2 N /(N-2)}\left(\mathbf{R}^{N}\right)$, the result follows with $u^{0 \#}=: u^{0}-u$.

\section{The auxiliary problem}

We are in a position to be more precise about the aforementioned necessary result, see section 7 .

Proposition 8.1 The limit $u^{0}$ solves the following boundary value problem

$$
\begin{gathered}
-\gamma \Delta_{y} u^{0}+\frac{u^{0}}{|D|}=0, \quad \text { in } \Omega \times D \\
-\gamma \Delta_{y} u^{0}=0 \quad \text { in } \Omega \times \mathbf{R}^{N} \backslash D \\
{\left[u^{0}\right]_{\partial D}=\left[\frac{\partial u^{0}}{\partial n}\right]_{\partial D}=0} \\
u^{0}-u \in L^{2}\left(\Omega ; L^{2 N /(N-2)}\left(\mathbf{R}^{N}\right)\right) \cap L^{2}\left(\Omega ; H^{1}\left(\mathbf{R}^{N}\right)\right) .
\end{gathered}
$$

Proof Let $\phi \in C_{c}^{1}\left(\mathbf{R}^{N}\right)$ and let $\psi \in \mathcal{D}(\Omega)$. Then, taking $\Phi^{\varepsilon}=\phi\left(y^{\varepsilon}\right) N_{\varepsilon}(\psi)$ as a test function in the variational formulation (55), we get

$$
\int_{\Omega} \nabla u^{\varepsilon} \nabla \Phi^{\varepsilon}+f_{D_{\varepsilon}} u^{\varepsilon} \Phi^{\varepsilon}=\int_{\Omega} f^{\varepsilon} \Phi^{\varepsilon}
$$

with

$$
\begin{aligned}
\int_{\Omega} \nabla u^{\varepsilon} \nabla \Phi^{\varepsilon} & =\int_{\Omega} \nabla u^{\varepsilon} \nabla\left(\phi\left(y^{\varepsilon}\right)\right) N_{\varepsilon}(\psi)=\frac{1}{r_{\varepsilon}} \int_{\Omega} \nabla u^{\varepsilon} \nabla_{y} \phi\left(y^{\varepsilon}\right) N_{\varepsilon}(\psi) \\
& =\frac{1}{r_{\varepsilon}^{2}} \int_{\Omega}\left(f_{K_{\varepsilon}} \nabla_{y} \tilde{u}^{\varepsilon} \nabla_{y} \phi(y)\right)=\gamma_{\varepsilon} \int_{\Omega \times K_{\varepsilon}} \nabla_{y} \tilde{u}^{\varepsilon} \nabla_{y} \phi \\
& \rightarrow \gamma \int_{\Omega \times \mathbf{R}^{N}} \psi \nabla_{y} u^{0} \nabla_{y} \phi=\gamma \int_{\Omega}\left(\int_{\mathbf{R}^{N}} \nabla_{y} u^{0} \nabla_{y} \phi\right), \\
f_{D_{\varepsilon}} u^{\varepsilon} \Phi^{\varepsilon} & =f_{D_{\varepsilon}} u^{\varepsilon} \phi\left(y^{\varepsilon}\right) N_{\varepsilon}(\psi)=\frac{1}{\varepsilon^{N} \operatorname{card} \mathbf{Z}_{\varepsilon}} f_{\Omega \times D} \tilde{u}^{\varepsilon} \phi(y) \psi \rightarrow \int_{\Omega}\left(f_{D} u^{0} \phi(y)\right) .
\end{aligned}
$$

Moreover, we note that

$$
\phi\left(y^{\varepsilon}\right) N_{\varepsilon}(\psi) \rightarrow 0 \quad \text { in } H_{0}^{1}(\Omega) .
$$


Indeed, assuming that $\operatorname{supp} \phi \subset B(0, R)$ for some $R>0$, and noticing that

$$
\left|y^{\varepsilon}\right| \leq R \Longleftrightarrow\left|x-\varepsilon\left[\frac{x}{\varepsilon}\right]\right| \leq r_{\varepsilon} R,
$$

we get

$$
\begin{aligned}
\left|\phi\left(y^{\varepsilon}\right) N_{\varepsilon}(\psi)\right|_{\Omega} & \leq C|\phi|_{\infty}|\psi|_{\infty} \frac{|\Omega|}{\varepsilon^{N}}\left(r_{\varepsilon} R\right)^{N}=C\left(\frac{r_{\varepsilon} R}{\varepsilon}\right)^{N} \rightarrow 0 \\
\left|\nabla\left(\phi\left(y^{\varepsilon}\right) N_{\varepsilon}(\psi)\right)\right|_{\Omega} & =\left|\frac{1}{r_{\varepsilon}} \nabla_{y} \phi\left(y^{\varepsilon}\right) N_{\varepsilon}(\psi)\right|_{\Omega} \leq\left|\nabla_{y} \phi\right|_{\infty}|\psi|_{\infty} \frac{C}{r_{\varepsilon}}\left(\frac{r_{\varepsilon} R}{\varepsilon}\right)^{N}=C r_{\varepsilon} \gamma_{\varepsilon} R^{N} \rightarrow 0
\end{aligned}
$$

which yields (72). We deduce that

$$
\int_{\Omega} f^{\varepsilon} \Phi^{\varepsilon}=\int_{\Omega^{\varepsilon}} f^{\varepsilon} \phi\left(y^{\varepsilon}\right) N_{\varepsilon}(\psi) \rightarrow 0 .
$$

There results

$$
\gamma \int_{\Omega}\left(\int_{\mathbf{R}^{N}} \nabla_{y} u^{0} \nabla_{y} \phi\right)+\int_{\Omega}\left(f_{D} u^{0} \phi(y)\right)=0 .
$$

The analog of Theorem 5.4 can be established: see in particular formula (48).

Proposition 8.2 The application $v$ defined in Proposition 3.1 can be identified as

$$
v=u\left(1-f_{D} \chi^{0}\right)
$$

where:

$$
\chi^{0} \in L^{2 N /(N-2)}\left(\mathbf{R}^{N}\right) \cap H^{1}\left(\mathbf{R}^{N}\right)
$$

is the unique solution of (38)-(40).

Proof Let $\varphi \in \mathcal{D}(\Omega)$. Then, Proposition 3.1 still holds true and we have:

$$
\int_{\Omega} v \varphi=\lim _{\varepsilon \rightarrow 0} \int_{D_{\varepsilon}} u^{\varepsilon} \varphi
$$

Note that from the Mean value theorem

$$
\left|N_{\varepsilon}(\varphi)-\varphi\right|_{\infty} \leq 2 \varepsilon|\nabla \varphi|_{\infty}
$$


which yields

$$
\left|f_{D_{\varepsilon}} u^{\varepsilon} \varphi-f_{D_{\varepsilon}} u^{\varepsilon} N_{\varepsilon}(\varphi)\right| \leq C \varepsilon f_{D_{\varepsilon}}\left|u^{\varepsilon}\right||\nabla \varphi|_{\infty} \leq C \varepsilon\left(f_{D_{\varepsilon}}\left|u^{\varepsilon}\right|^{2}\right)^{1 / 2}|\nabla \varphi|_{\infty} \leq C \varepsilon
$$

There results

$$
\int_{\Omega} v \varphi=\lim _{\varepsilon \rightarrow 0} f_{D_{\varepsilon}} u^{\varepsilon} N_{\varepsilon}(\varphi)=\lim _{\varepsilon \rightarrow 0} f_{\Omega \times D} \tilde{u}^{\varepsilon} \varphi=f_{\Omega \times D} u^{0} \varphi
$$

that is,

$$
v=f_{D} u^{0}
$$

To conclude, note that the limit $u^{0}$ solves the following boundary value problem:

$$
\begin{gathered}
-\gamma \Delta_{y} u^{0}+\frac{u^{0}-u}{|D|}=\frac{-u}{|D|}, \quad \text { in } \Omega \times D \\
-\gamma \Delta_{y} u^{0}=0 \quad \text { in } \Omega \times \mathbf{R}^{N} \backslash D \\
{\left[u^{0}\right]_{\partial D}=\left[\frac{\partial u^{0}}{\partial n}\right]_{\partial D}=0} \\
u^{0}-u \in L^{2}\left(\Omega ; L^{2 N /(N-2)}\left(\mathbf{R}^{N}\right)\right) \cap L^{2}\left(\Omega ; H^{1}\left(\mathbf{R}^{N}\right)\right) .
\end{gathered}
$$

Due to the linearity of the problems (76)-(79) and the unicity of its solution for any given $u$, we infer that $u^{0}-u$ splits as

$$
u^{0}-u=-u \chi^{0}
$$

where $\chi^{0}$ solves the problems (38)-(40), (74) which does not depend on $u$. Then, the conclusion comes thanks to (75).

Remark 1 We conclude as in the proof of Theorem 5.4 using the energy method (49)-(51) to get $v=h$.

\section{References}

[1] Bellieud, M. and Gruais, I., 2005, Homogenization of an elastic material reinforced by very stiff or heavy fibers. Non local effects. Memory effects. Journal of Mathematiques Pures et Applquées, 84(1), 55-96.

[2] Bentalha, F., Gruais, I. and Polisevski, D., 2006, Homogenization of a conductive suspension in a Stokes-Boussinesq flow. Applicable Analysis, 85(6/7), 811-830.

[3] Bentalha, F., Gruais, I. and Polisevski, D., 2007, Diffusion process in a rarefied binary structure (To appear in Revue Roumaine de Mathématiques Pures et Appliquées).

[4] Bentalha, F., Gruais, I. and Polisevski, D., 2006, Diffusion in a highly rarefied binary structure of general periodic shape. Prépublication de l'I.R.M.A.R.

[5] Brezis, H., 1983, Analyse Fonctionelle. Théorie et Applications (Paris: Masson).

[6] Gruais, I., 2006, Homogenization and mixed scales in fluids. Revue Roumaine de Mathématiques Pures et Appliquées, 51(3), 311-329. 


\section{Appendix A: a maximum principle}

An adaptation of Theorem IX.27 [5] leads to the following result.

Proposition A1 For every $\lambda>1$, the solution $\chi \lambda$ of the problems (28)-(31) satisfies the following maximum principle:

$$
0 \leq \chi \lambda \leq 1 \quad \text { in } B_{\lambda}
$$

Proof Using Stampacchia's truncature, we let $G \in \mathcal{C}^{1}(\mathbf{R})$ satisfying

$$
\begin{gathered}
\left|G^{\prime}(s)\right| \leq M, \quad \forall s \in \mathbf{R} \\
G \text { is strictly increasing in }] 0,+\infty[ \\
G(s)=0, \quad \forall s \leq 0 .
\end{gathered}
$$

Let $\zeta_{\lambda}=G(\chi \lambda-1)$. Then, $\zeta_{\lambda} \in H^{1}\left(B_{\lambda}\right)$ and Dirichlet's condition (31) together with the assumption (A3) on $G$ lead to $\left.\zeta_{\lambda}\right|_{\partial B_{\lambda}}=0$ and therefore $\zeta_{\lambda} \in H_{0}^{1}\left(B_{\lambda}\right)$. Choosing $\zeta_{\lambda}$ as a test function in the variational formulation of (28)-(31), we get

$$
\int_{B_{\lambda}}\left|\nabla_{y} \chi \lambda\right|^{2} G^{\prime}(\chi \lambda-1)+f_{D}(\chi \lambda-1) G(\chi \lambda-1)=0 .
$$

The first term in the left-hand side of (A4) is nonnegative because of (A1). As for the second term, the integral actually reduces to an integral defined on the subset of $D$ where $\chi \lambda-1 \geq 0$ and it is thus nonnegative. There follows that (A4) is vanishing while at the same time being the sum of nonnegative terms, which implies that each of its terms is zero:

$$
\int_{B_{\lambda}}\left|\nabla_{y} \chi \lambda\right|^{2} G^{\prime}(\chi \lambda-1)=f_{D}(\chi \lambda-1) G(\chi \lambda-1)=0 .
$$

Finally, $(\chi \lambda-1) G(\chi \lambda-1)=0$ a.e. in $D$. If we assume that $\chi \lambda-1 \neq 0$ in some subset $A \subset D$ with positive Lebesgue measure, then, for every $y \in A, G(\chi \lambda(y)-1)=0$, and thus, taking into account (A2)-(A3), we find that $\chi \lambda<1$ in $A$, a contradiction. Finally: $\chi_{\lambda} \leq 1$ a.e. in $D$, which is the first part of the proposition. The same arguments with $\chi \lambda$ and $\zeta_{\lambda}$ replaced by $-\chi \lambda$ and $G(-\chi \lambda)$ respectively show that $-\chi \lambda \leq 0$ a.e. in $D$, and the proof is complete. 